\title{
Floating-gate based PN blending optoelectronic synaptic transistor for neural machine translation
}

\author{
Xianghong Zhang ${ }^{1,2}$, Enlong $\mathrm{Li}^{1,2}$, Rengjian $\mathrm{Yu}^{1,2}$, Lihua $\mathrm{He}^{1,2}$, Weijie $\mathrm{Yu}^{1,2}$, Huipeng $\mathrm{Chen}^{1,2^{*}}$ and \\ Tailiang Guo ${ }^{1,2}$
}

\begin{abstract}
Neural machine translation, which has an encoder-decoder framework, is considered to be a feasible way for future machine translation. Nevertheless, with the fusion of multiple languages and the continuous emergence of new words, most current neural machine translation systems based on von Neumann's architecture have seen a substantial increase in the number of devices for the decoder, resulting in high-energy consumption rate. Here, a multilevel photosensitive blending semiconductor optoelectronic synaptic transistor (MOST) with two different trapping mechanisms is firstly demonstrated, which exhibits 8 stable and well distinguishable states and synaptic behaviors such as excitatory postsynaptic current, short-term memory, and long-term memory are successfully mimicked under illumination in the wavelength range of $480-800 \mathrm{~nm}$. More importantly, an optical decoder model based on MOST is successfully fabricated, which is the first application of neuromorphic device in the field of neural machine translation, significantly simplifying the structure of traditional neural machine translation system. Moreover, as a multi-level synaptic device, MOST can further reduce the number of components and simplify the structure of the codec model under light illumination. This work first applies the neuromorphic device to neural machine translation, and proposes a multi-level synaptic transistor as the based cell of decoding module, which would lay the foundation for breaking the bottleneck of machine translation.
\end{abstract}

Keywords: optoelectronic transistor, synaptic transistor, synaptic plasticity modulation, neural machine translation, decoder

\section{INTRODUCTION}

One of the major goals of artificial intelligence is that machine translation systems can reach the human level [1], while the level of machine translation is now still far below the human level. At present, the main bottlenecks that limit machine translation is the high-energy consumption rate of decoder (Fig. 1a), a crucial part of the most popular translation model-neural machine translation (NMT) (Fig. S1) [2-4]. With the fusion of multiple languages and the continuous emergence of new words, the calculation results of the NMT model have become more and more complex [5], and the number of components required for decoding has increased rapidly, which results in a slow-decoding speed and high-energy consumption. In order to alleviate the difficulty of decoding, significant efforts have been devoted to the optimization of algorithms (such as importance sampling and monolingual data to speed up translation) and tremendous progress has been made in the software [6-10], while little attention has been paid to hardware which is equally important for the development of decoding. Due to the separation between the storage and processing units, the conventional computers based on the traditional von Neumann architecture extremely limit the development of highly efficient computing and decoding [11]. Meanwhile, it requires multiple complementary metal oxide semiconductors (CMOSs) to complete the basic operations (Fig. 1b), but subject to Moore's Law, the density of devices on the same chip area will increase with the increase of data. The current microelectronics manufacturing process will soon reach the physical limit of materials and cannot keep providing support for the development of Moore's Law [12].

To break through the limitations of the von Neumann's architecture and Moore's Law, tremendous attention has been paid to the neuromorphic architecture $[13,14]$, which has provided a promising paradigm for data storage, thus eliminating the cost of data transfer $[11,15,16]$ by imitating biological brains. The synaptic device with tunable resistance is the basic cell of neuromorphic devices [17], and many kinds of synaptic devices such as two-terminal memristors [18-20], phase-change memories $[21,22]$ and three-terminal field-effect synaptic transistors have already been demonstrated [23-26]. Compared with CMOS-based synaptic devices, these novel synaptic devices have advantages as follows: (i) they can achieve synaptic functions through a single device, which reduces energy consumption. (ii) Their active layer is simple in design. (iii) They can be prepared by simple processes such as the solution method and printing, which is conducive to large-scale array production. In addition, the three-terminal synaptic transistor, compared with the two-terminal one, achieves read-write separation and has the ability for spatiotemporal information processing, and is regarded as one of the best candidates for the establishment of neuromorphic devices [27-29]. Unfortunately, synaptic transistor is mostly utilized in the field of bionic devices [30-32], smart sensing [33-35], image processing and pattern recognition [3638], whereas there are no reports about its application in the field of NMT.

In this work, the neuromorphic devices are firstly used as the decoder of NMT (Fig. 1c), where a multilevel photosensitive blending semiconductor optoelectronic synaptic transistor

\footnotetext{
${ }^{1}$ Institute of Optoelectronic Display, National \& Local United Engineering Lab of Flat Panel Display Technology, Fuzhou University, Fuzhou 350002, China

${ }^{2}$ Fujian Science \& Technology Innovation Laboratory for Optoelectronic Information of China, Fuzhou 350100, China

* Corresponding author (email: hpchen@fzu.edu.cn)
} 
a

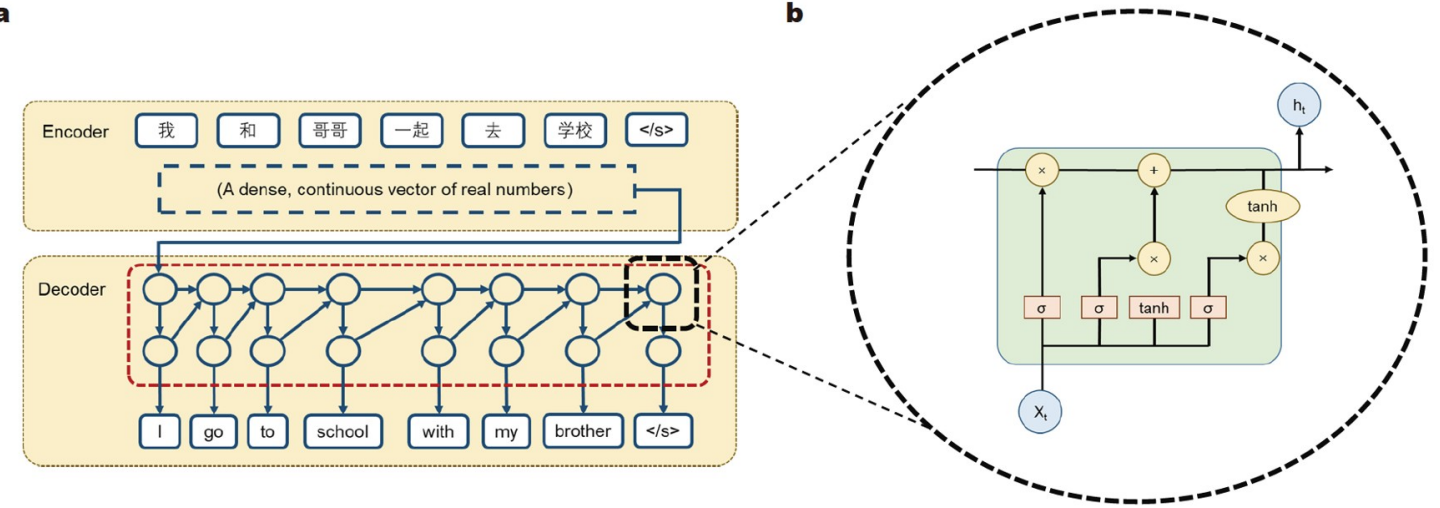

c

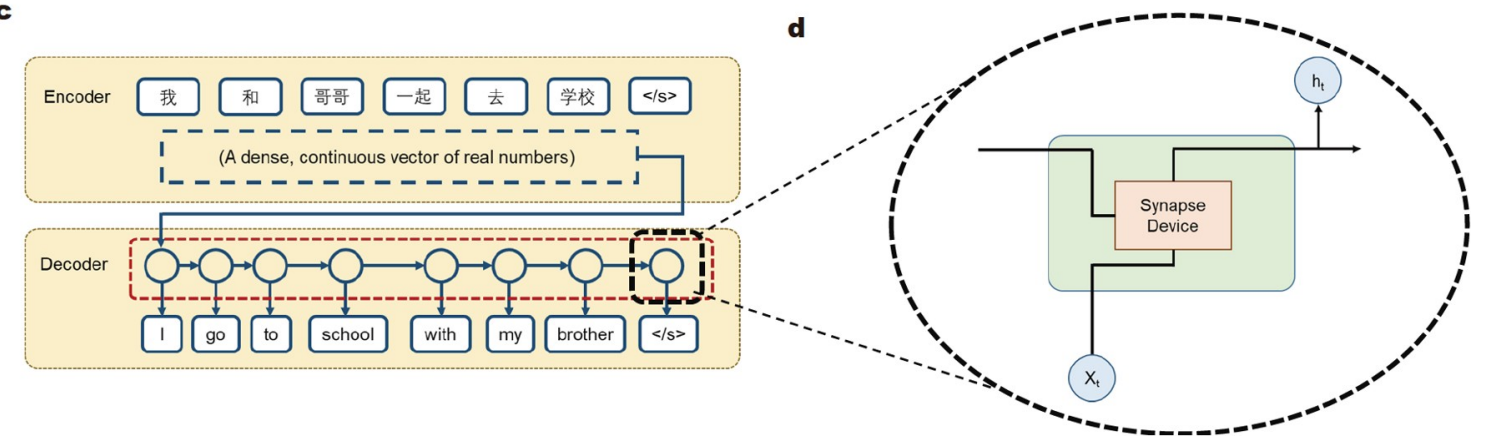

Figure 1 (a) The traditional encoder-decoder framework of NMT. (b) The single kernel of the traditional encoder-decoder model. (c) The encoder-decoder framework with neuromorphic architecture. (d) The single kernel of the encoder-decoder model based on the synaptic device.

(MOST) with two different trapping mechanisms along with 8 stable and well distinguishable states is proposed as the decoding module for NMT. Synaptic behaviors such as excitatory postsynaptic current (EPSC), short-term memory (STM), long-term memory (LTM) are successfully mimicked. Based on MOST, an optical decoder model (ODM) is then fabricated, which can replace the basic node composed of multiple CMOS in traditional NMT with a single synaptic device (Fig. 1d), significantly simplifying the structure of traditional NMT system. Moreover, as a multi-level synaptic device, MOST can further reduce the number of components and simplify the structure of the model under light illumination (Fig. S2). Therefore, this work proposes a synaptic transistor-based decoding module, which will lay the foundation for breaking the bottleneck of machine translation in the future.

\section{EXPERIMENTAL SECTION}

\section{Device fabrication}

Indacenodithiophene-benzothiadiazole (IDTBT) and [6,6]-phenyl C61 butyric acid methyl ester $\left(\mathrm{PC}_{61} \mathrm{BM}\right)$ (the chemical structures of them are shown in Fig. S3) were purchased from Derthon Optoelectric Materials Science Technology Co. Ltd. and used without further purification. The non-volatile organic memory device was fabricated on a heavily doped n-type Si wafer with a 100 -nm-thick thermally grown $\mathrm{SiO}_{2}$ gate dielectric layer. IDTBT and $\mathrm{PC}_{61} \mathrm{BM}$ were dissolved in chloroform (molar ratio of IDTBT: $\mathrm{PC}_{61} \mathrm{BM}=5: 1$ ). The substrate was cleaned by sonication in acetone, isopropanol, and deionized water for 10 min each. In order to better disperse the CdSe@ZnS quantum dots (QDs), they were mixed in cross-linked polyvinylpyrrolidone (PVP) with a mass ratio of 1:10. The mixed solution was deposited on a $\mathrm{Si}$ wafer with $100 \mathrm{~nm} \mathrm{SiO}$ by spincoating at $600 \mathrm{r} \mathrm{min}^{-1}$ for $5 \mathrm{~s}$ and $2000 \mathrm{r} \mathrm{min}^{-1}$ for $30 \mathrm{~s}$, and then annealed at $120^{\circ} \mathrm{C}$ for $2 \mathrm{~h}$ in a nitrogen environment. Then, the mixed semiconductor layer of IDTBT:20\% $\mathrm{PC}_{61} \mathrm{BM}$ was spincoated on the top of the QD layer at $1000 \mathrm{r} \mathrm{min}^{-1}$ for $60 \mathrm{~s}$, and then annealed at $150^{\circ} \mathrm{C}$ for $10 \mathrm{~min}$. Finally, 50-nm $\mathrm{Au}$ was deposited as source and drain electrodes by thermal evaporation, and the entire surface of $\mathrm{Au}$ was obtained without using a mask when fabricating the array. Laser generated by an ultrafast Ti: sapphire amplifier system (Coherent Astrella, pulse duration $45 \mathrm{fs}$, center wavelength $800 \mathrm{~nm}$, repetition rate $1 \mathrm{kHz}$ ) was employed to etch Au to obtain the MOST-based array (Fig. S4). The array was connected to the analog to digital converter (ADC) module and microcontroller unit (MCU) module through conductive silver paste (annealed at $100^{\circ} \mathrm{C}$ for $5 \mathrm{~min}$ ) and displayed on the personal computer (PC) terminal (Fig. S5).

\section{Device characterization}

The electrical characteristics and basic synaptic functions were measured in the surrounding environment with a semiconductor parameter analyzer (Keithley 4200-SCS). The ultraviolet-visible absorption spectrum was measured using an ultraviolet-visible-near infrared spectrophotometer (Shimadzu UV-3600 Plus).

\section{Simulation}

The long short-term memory (LSTM) layer was simulated by Matlab after collecting the hardware output data.

\section{RESULTS AND DISCUSSION}

The neuromorphic structure, which is a network composed of numerous synapses and neurons, provides an energy-efficient 
solution for complicated compute tasks such as big data and nonlinear calculation. As one of the fundamental units, synaptic devices are mainly employed to transmit and integrate the processed signal between neurons [29]. The key step in applying the neuromorphic structure to NMT is imitating the process, which changes the external input information to induce related neurons in the language center of the biological brain to generate neural signals that are processed through synapses. In this work, electrical and light signals are used as input signals, the multilevel photosensitive blending MOST is used as a synaptic device, and changes of drain current in MOST represent changes in neural signals caused by different input information (Fig. 2a). The MOST is composed of QDs and IDTBT-mixed $\mathrm{PC}_{61} \mathrm{BM}$ with a bottom-gate/top-contact structure, as presented in Fig. 2b. The PVP-mixed CdSe@ZnS QDs are served as a floating gate layer to capture the carriers generated by electrons. The blending semiconductor of IDTBT and $\mathrm{PC}_{61} \mathrm{BM}$ mixed in a ratio of 5:1 acts as a light absorption layer. The absorption spectrum of the mixed semiconductor material is presented in Fig. 2c, which shows that the absorption of light in the wavelength range of $480-700 \mathrm{~nm}$ is strong with weak absorption in $700-800 \mathrm{~nm}$. $\mathrm{Au}(50 \mathrm{~nm})$ is used as the source/drain contact electrode (the channel length is $\sim 30 \mu \mathrm{m}$ and the width is $\sim 500 \mu \mathrm{m}$ ), and detailed information about the fabrication of the device is described in the EXPERIMENTAL SECTION.

The fundamental photo-transistor characteristics of MOST were firstly examined. The dual-swept transfer curves (from 40 to $-40 \mathrm{~V}$ ) under illumination with different wavelengths are shown in Fig. 3a, which exhibit the behavior of p-type transistor with an on/off current ratio $\left(I_{\mathrm{ON}} / I_{\mathrm{OFF}}\right)$ greater than $10^{4}$. The $I_{\mathrm{ON}} /$ $I_{\mathrm{OFF}}$ remains almost constant under illumination with different wavelengths, while it positively moves the threshold voltage. MOST has a large counterclockwise $I_{\mathrm{DS}}-V_{\mathrm{GS}}$ hysteresis window, with the values of about $39.5,41.2,44.3$ and $46.1 \mathrm{~V}$ in the dark, 525,600 and $675 \mathrm{~nm}$ environments, respectively (Fig. S6). Synaptic behavior can be observed in the device, where the gate electrode and light are utilized as two pre-synapses, the drain electrode is considered as post-synapse, the carriers in the channel of the device are neurotransmitters, and the change of device conductance after pulse represents the connection strength of the front and post neurons. When applying a fixed voltage pulse $\left(V_{\mathrm{GS}}=5 \mathrm{~V}, t_{\text {pulse }}=30 \mathrm{~ms}, V_{\mathrm{DS}}=-20 \mathrm{~V}\right)$ (Fig. $\left.3 \mathrm{~b}\right)$, it can be found that the EPSC after the pulse changes with the light wavelengths. In the dark condition, EPSC will quickly decay to the value that is slightly higher than the initial value (the curve labelled 'Dark' in Fig. 3b), while EPSC attenuation becomes slower under light exposure (the curve labelled ' $525 \mathrm{~nm}$ ' ' $600 \mathrm{~nm}$ ' and ' $680 \mathrm{~nm}$ ' in Fig. 3b). In addition, a similar phenomenon can also be observed when the amplitude of the electric pulse is changed under dark (Fig. S7), which shows that an increase of the amplitude of the input electrical pulse will reduce the EPSC attenuation $\left(V_{\mathrm{GS}}=10,30\right.$ and $40 \mathrm{~V}, t_{\text {pulse }}=$ $\left.30 \mathrm{~ms}, V_{\mathrm{DS}}=20 \mathrm{~V}\right)$. This change in EPSC is similar to the plasticity of biological synapses, which can form different

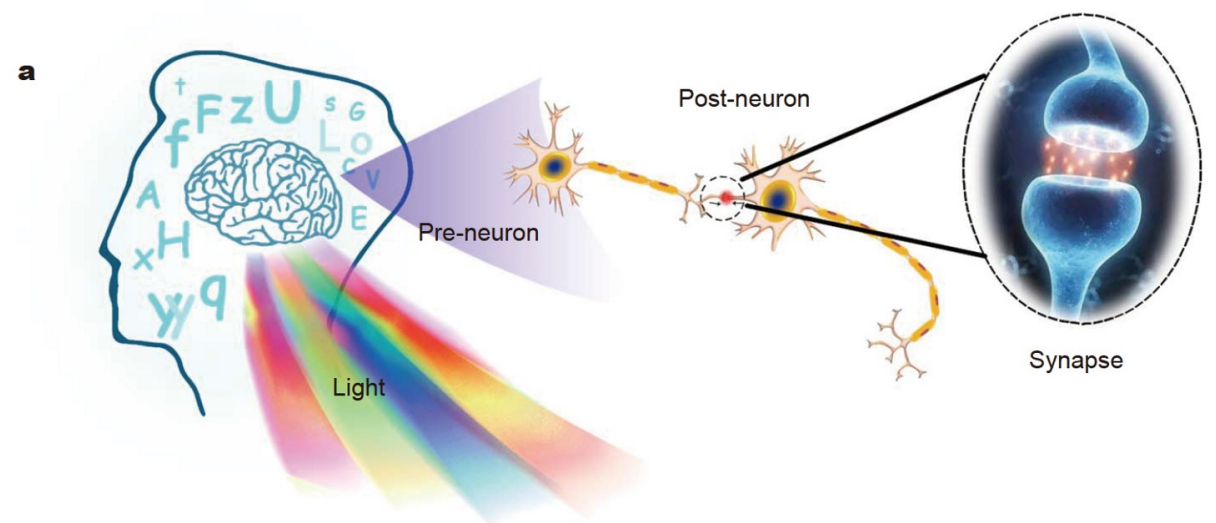

b

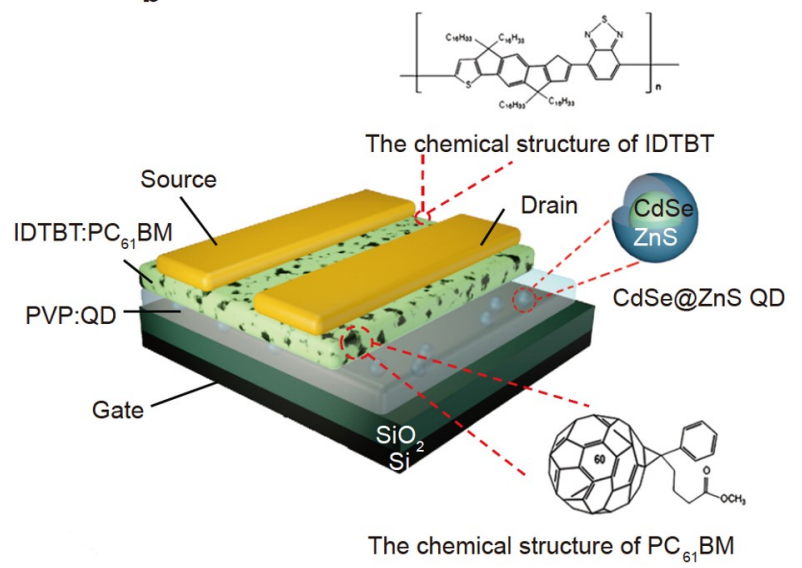

c

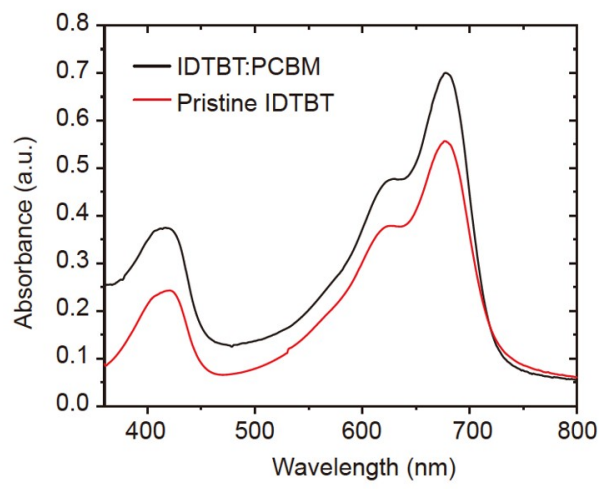

Figure 2 (a) Schematic of biology of the brain, neuron cell and synapses. (b) Schematic diagram of the device architecture of the MOST. (c) The absorption of IDTBT:PC ${ }_{61} \mathrm{BM}$ and IDTBT. 

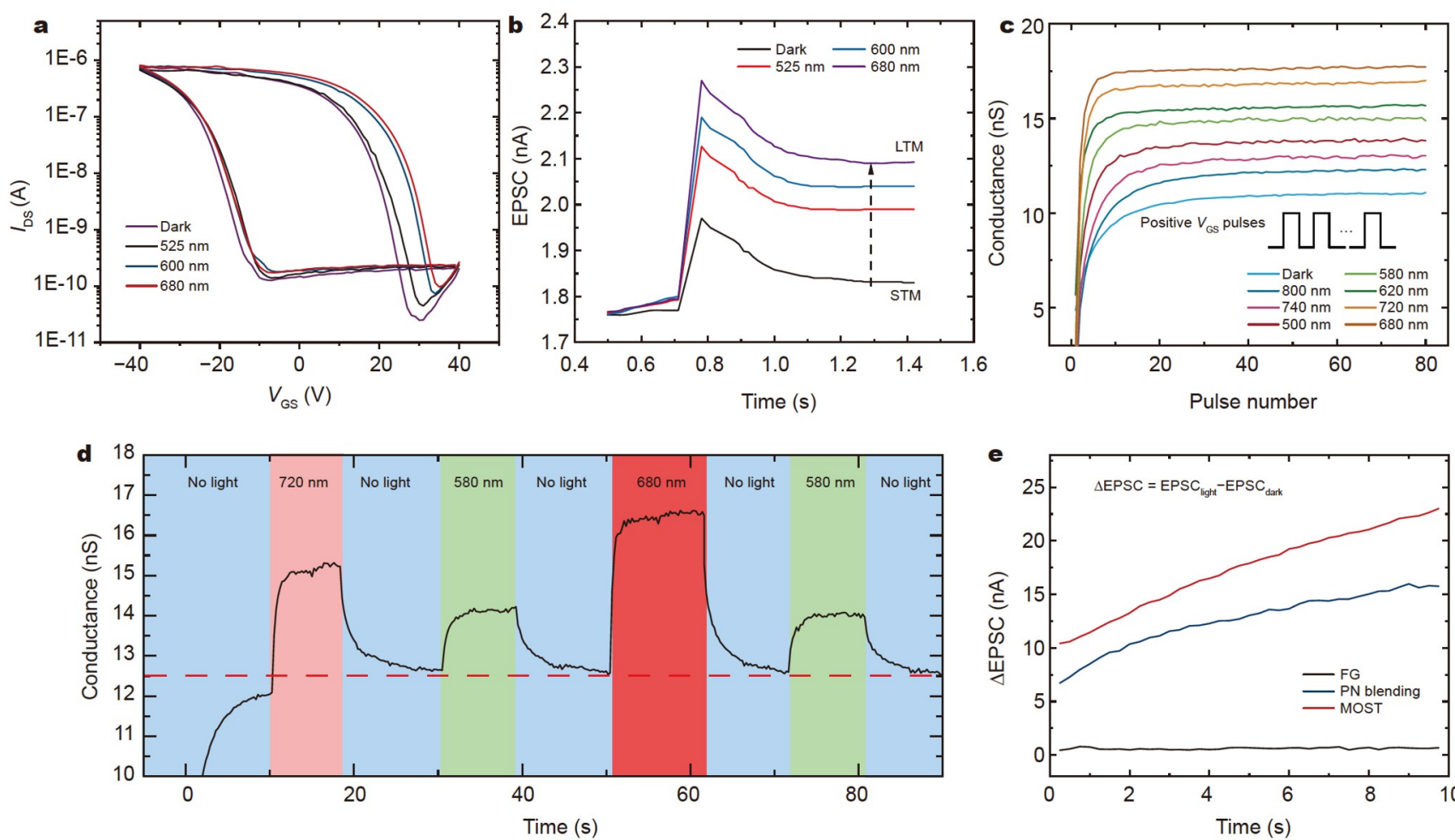

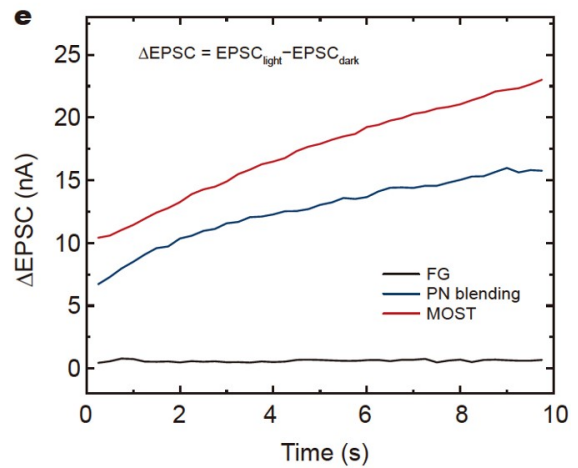

Figure 3 (a) Transfer curves of the MOST measured in double sweep under illumination with wavelengths of 525 , 600 , and $680 \mathrm{~nm}$, at $V_{\mathrm{DS}}=-20 \mathrm{~V}$. (b) EPSC triggered by a presynaptic voltage pulse $\left(V_{\mathrm{GS}}=5 \mathrm{~V}, t_{\text {pulse }}=30 \mathrm{~ms}\right)$ as a function of time under illumination with wavelengths of 525 , 600 , and $680 \mathrm{~nm}$. (c) EPSC stimulated by a series of $5 \mathrm{~V}$ pulses with a duration of $30 \mathrm{~ms}$ under illumination with wavelengths of $500,580,620,680,720,740$ and $800 \mathrm{~nm}$. (d) EPSC stimulated by a series of $5 \mathrm{~V}$ pulses by changing the wavelength of the irradiated light. (e) The $\triangle \mathrm{EPSC}$ of MOST, PN blending synaptic transistor and FG synaptic transistor by 40 consecutive $5 \mathrm{~V}$ pulses.

degrees of memory through different stimuli. Under dark, the device exhibits STM through the stimulation of electric pulses with small amplitude, and changing the light conditions or the amplitude of the input electric pulses will cause the device to form LTM. The above behavior is the basis for imitating the calculation process of the biological brain. In addition, in the biological brain, the repeated action of the same stimulus will also enable the device to obtain LTM. As shown in Fig. $3 \mathrm{c}$ and Fig. S8, the conductance of MOST increases significantly before 15 voltage pulses stimulated and will not show a saturation trend until 20 voltage pulses applied, and more results are shown in Fig. S8. The conductance of 50 MOSTs after 20 voltage pulses are counted in Fig. S9, which indicates that the conductance remains at different stable values, and the difference in conductance is about $1 \mathrm{nS}$ under different illuminations. The results demonstrate that the device achieves different LTM after 20 voltage pulses stimulated under different illuminations, and MOST can be controlled by light to work stably at different synaptic levels. Moreover, under continuous pulses, after the light is removed, the conductance value of the device will slowly decay to the saturation conductance value in the dark, and then reapplying illumination will cause the conductance value of the device change again. To investigate the repeatability of MOST, we continuously use different wavelengths of light to irradiate the device and then remove the light (Fig. 3d). The results demonstrate that after continuously using different wavelengths of light to irradiate the device, the conductance value of the device will return to the same state after the light is removed. We believe that the above two phenomena have successfully imitated the potentiation and depression behaviors of biological synapses.

In order to evaluate the ability for multi-level synaptic device, the performance of three different synaptic devices, $\mathrm{p}$-type and n-type semiconductors (PN) blending synaptic transistor, floating-gate (FG) synaptic transistor and MOST, under photoelectric coordinated input was systematically investigated. Fig. S10 shows the effect of the concentration of the n-type semiconductor on MOST characteristics and the results indicate that the $I_{\mathrm{ON}} / I_{\mathrm{OFF}}$ decreases with the addition of a large amount of n-type semiconductor. Moreover, the effect of the concentration of QDs was investigated, and the results are shown in Fig. S11. It is shown that an FG transistor based on QDs can separate the photo-generated carriers, resulting in the shift of the transfer curve to the positive direction, while the concentration of QDs has a weaker effect on the amplitude of the curve movement. Fig. S12 shows the EPSC response during the application of 40 consecutive positive $V_{\mathrm{Gs}}$ pulse to three different synaptic devices under dark and light (wavelength $=680 \mathrm{~nm}, P=50 \mu \mathrm{W} \mathrm{cm} \mathrm{cm}^{-2}$ ). The change of EPSC $\left(\triangle E P S C=\right.$ EPSC $_{\text {light }}-$ EPSC $\left._{\text {dark }}\right)$ response is shown in Fig. 3e. The results show that EPSC is most affected by the light in MOST, indicating that MOST can obtain more well distinguished stable states under light than PN blend synaptic transistor and FG synaptic transistor.

On the basis of the characteristics of multi-level synaptic transistor, the operation mechanism of MOST is proposed and shown in Fig. 4. Firstly, the double swept transfer curve of IDTBT-based transistor is shown in Fig. S13, indicating that the IDTBT-based transistor without a big hysteresis window cannot work as a synaptic device. The energy level positions of IDTBT, 


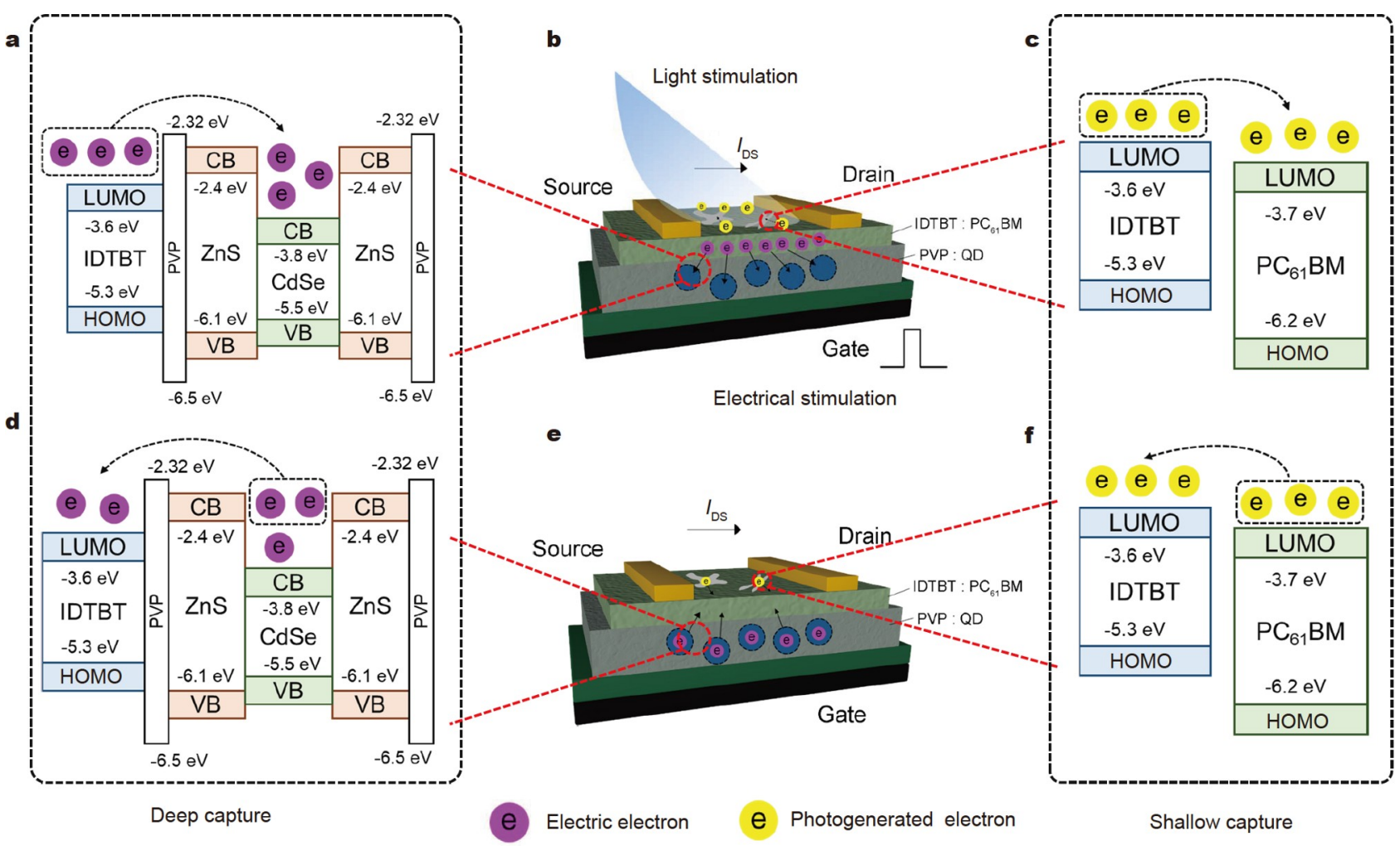

Figure 4 (a) The electric electrons will overcome the energy barrier to transfer from IDTBT to CdSe@ZnS when a positive $V_{\mathrm{GS}}$ is applied. HOMO: highest occupied molecular orbital; VB: valence band. (b) Under electrical and light stimulation, MOST has two different capture mechanisms. (c) Under the visible light, the photogenerated electrons will transfer to the discrete $\mathrm{PC}_{61} \mathrm{BM}$. (d) Partial electrons are trapped in CdSe@ZnS for a long time after removing $V_{\mathrm{GS}}$. (e) After removing the electrical and light stimulation, MOST has two different decapture mechanisms. (f) All photogenerated electrons in $\mathrm{PC}_{61} \mathrm{BM}_{\mathrm{M}}$ return to IDTBT after the light is removed.

$\mathrm{PC}_{61} \mathrm{BM}, \mathrm{PVP}$ and CdSe@ZnS in MOST are shown in Fig. 4a, c, which indicates the energy barrier between the lowest vacancy molecular orbital (LUMO) of IDTBT and $\mathrm{PC}_{61} \mathrm{BM}$ is too small to form the deep trap centers, while the energy barrier between the lowest conduction band (CB) of $\mathrm{ZnS}$ and CdSe could form deep trap centers. When a positive $V_{\mathrm{GS}}$ is applied to the device, the electric electrons at the interface of the semiconductor layer and the insulator have the chance to overcome the energy barrier to transfer to CdSe, and partial electrons are confined in the discontinuous n-type quantum well heterojunction composed of IDTBT/PC ${ }_{61} \mathrm{BM} / \mathrm{IDTBT}$, as shown in Fig. $4 \mathrm{a}$, b. The trapped electrons lead to an internal electric field and are coupled with the accumulation of holes in the semiconductor layer, resulting in the positive shift of transfer curves and EPSC. Due to the deep trap center formed by $\mathrm{CdSe}$ and $\mathrm{ZnS}$, partial electrons are trapped for a long time (Fig. $4 \mathrm{~d}, \mathrm{e}$ ), resulting in the conversion of STM to LTM after a series of positive $V_{\mathrm{GS}}$ pulses (Fig. 3c). When the device is illuminated under the visible light, IDTBT absorbs the photons and generates photoexcitation in the blend films. Since the interface area between IDTBT and $\mathrm{PC}_{61} \mathrm{BM}$ is larger than that between IDTBT and QDs, and IDTBT is directly contacted with $\mathrm{PC}_{61} \mathrm{BM}$, most photogenerated electrons are transferred to the discrete $\mathrm{PC}_{61} \mathrm{BM}$, which is responsible for the multiple synaptic levels under constant illumination (Fig. 4b, c). Due to the shallow trap center formed by IDTBT and $\mathrm{PC}_{61} \mathrm{BM}$, all photogenerated electrons return to IDTBT after the light is removed (Fig. 4c, f), resulting in the returning of the conductance value of the device to the same state (Fig. 3d). As shown in Fig. S14, the Kelvin probe force microscopy results demonstrate that MOST has the largest surface potential compared with PN blend synaptic transistor and FG synaptic transistor with $5 \mathrm{~V} \quad V_{\mathrm{GS}}$ supplying under the light of $680-\mathrm{nm}$ wavelength, which further verifies that there are two capture mechanisms for MOST when a positive $V_{\mathrm{GS}}$ is applied to the device under the visible light.

Due to the excellent photoelectric response along with multistates, MOST is then applied to NMT. NMT is usually based on an encoder-decoder framework and attempts to build a decoder with the trained neural network that inputs a source language and outputs the translation (Fig. 5a), and more information about the encoder-decoder framework is provided in the Supplementary information (S1) $[39,40]$. The core of the decoder is the LSTM layer, a special recurrent network, which can use historical information to process the context in the translation task. The LSTM layer sets the system output signal of the last time sequence as the system input signal of the current time sequence, and its calculation unit mimics the biological memory function to process data. The traditional LSTM layer has a chain structure formed by a calculation unit composed by four sigmoid layers, which are used to imitate the learning, memory and forgetting behaviors of the human brain (Fig. S15). To compose the hardware LSTM layer, traditional CMOS transistor needs a large-scale circuit to simulate the behavior of a single synapse, which is limited by a large circuit area, high power consumption and poor compatibility (Fig. S16). Interestingly, synaptic devices have the same features, indicating that the MOST can be used as 
a

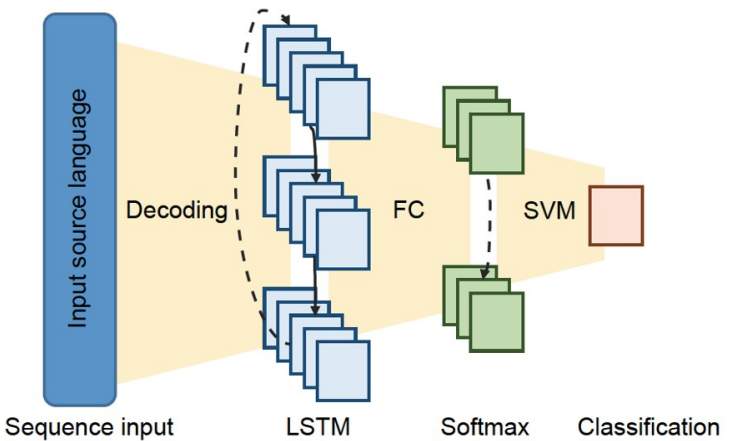

b

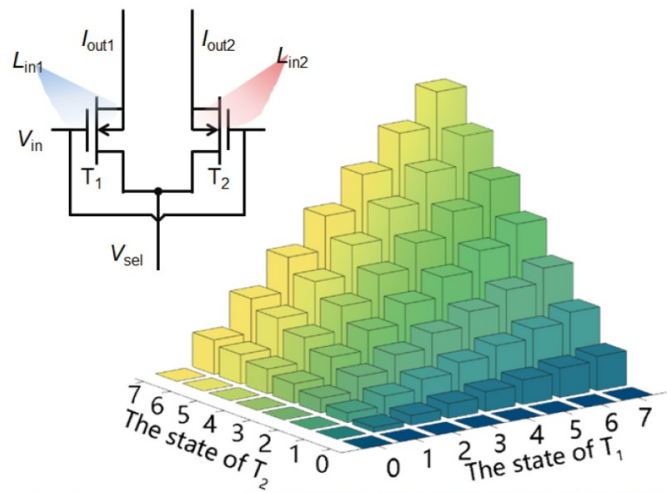

d c

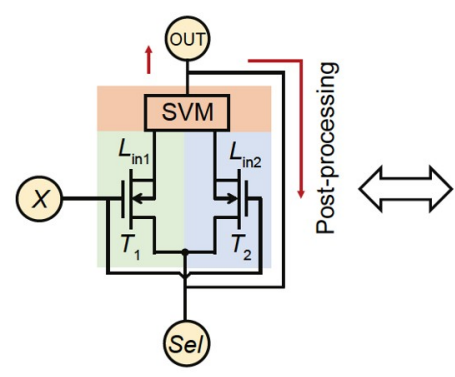

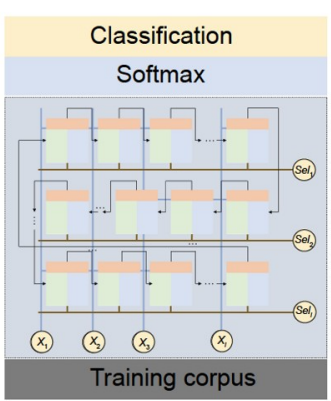

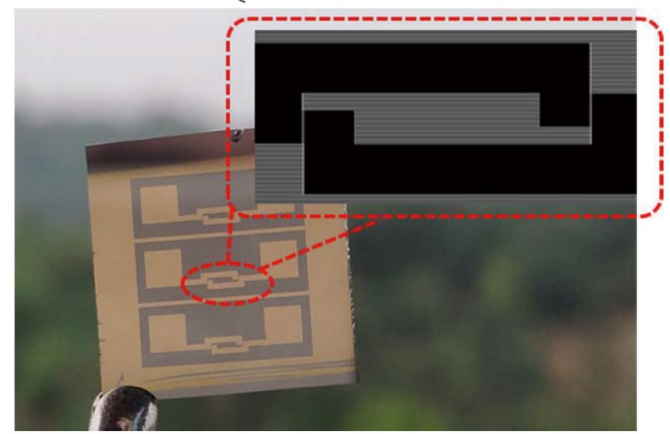

e
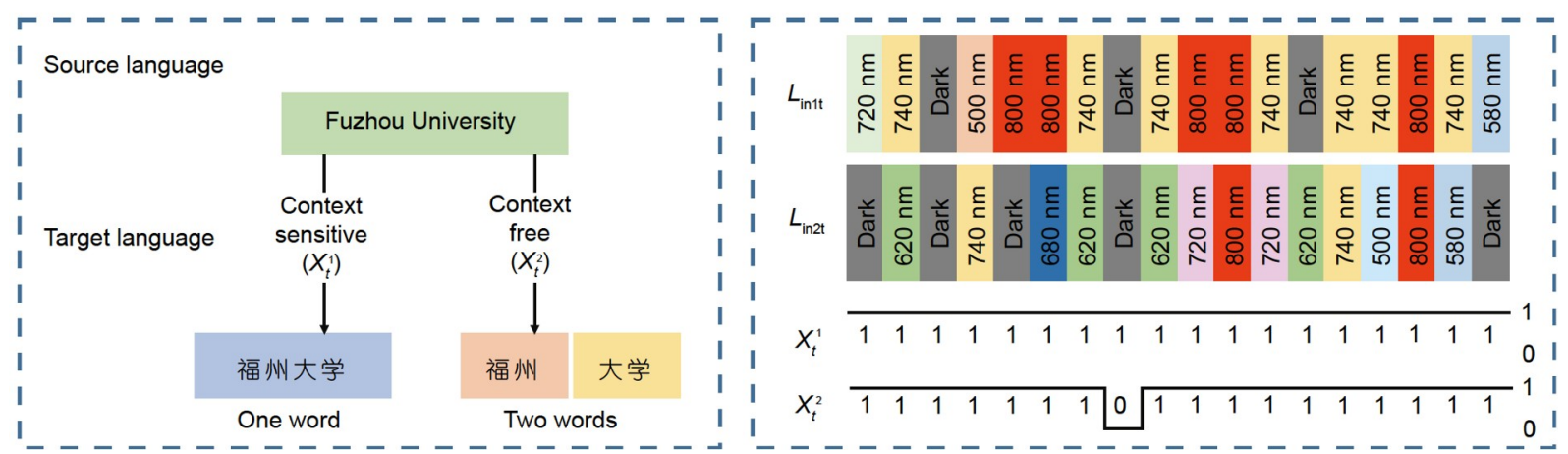

Figure 5 (a) LSTM recurrent neural network in an encoder-decoder framework. Fully Connected Layers (FC) is used to map feature information into the sample label space, and Support Vector Machines (SVM) is a two-class classification algorithm that can be used for linear classification and nonlinear classification. (b) The basic unit of the MOST-based classifier. (c) The schematic and framework of MOST-based LSTM networok. (d) MOST array for NMT. (e) Schematic illustration of the encoder-decoder model with MOST for NMT.

the based calculation unit of LSTM layer. The single-layer structure composed by MOST can replace the traditional foursigmoid-layer structure as the calculation unit of LSTM network, and significantly reduce the number of devices and eliminate the cost of data transfer, so that the power consumption is reduced to about $10^{-6} \mathrm{~W}$ [41]. Moreover, using multi-state synaptic devices further reduces the power consumption of the codec model, and alleviates the pressure caused by excessive training data (Fig. S17). Therefore, MOST as a multi-level synaptic device is applied to realize the learning and memory function of LSTM network, whose mathematical function is

$f(x)=\frac{a \times\left(x_{t-1}, x_{t}\right)}{1+b \times\left|x_{t-1}, x_{t}\right|}$,

where $f(x)$ is the result processed by MOST, $x_{t-1}$ is the memory left over from the previous input, $x_{t}$ is the current input, $a$ and $b$ are coefficients [42]. Under different wavelengths of light, MOST has different $a$ and $b$ coefficients as presented in
Table S1, indicating that MOST can be used as multiple synaptic devices, which greatly improves the data utilization rate of one device.

Here, we propose an optical decoder based on MOST to implement NMT tasks. As presented in Fig. 5b, two MOSTs are interconnected to form the smallest unit that distinguishes 64 different states, and detailed information about the fabrication of the device is described in the EXPERIMENTAL SECTION. MOST has 8 different states under different lighting (states $0-7$ as shown in Fig. 5b), and two MOSTs have 64 different states by co-connecting the gate and source electrode, where the two drain electrodes act as the output, which can input 64 different source language signs (include uppercase and lowercase letters, $0-9$, plus and slash; the conversion tables are presented in Table S2). The source language codes light sequences of different wavelengths as input to MOST, and $V_{\mathrm{GS}}$ controls whether to memorize the input at the previous moment (Logic 1 means memory, and logic 0 means forgetting). As presented in Fig. 5c, 
the principle of the basic unit of decoder based on MOST is

Out $_{t}=f\left(L_{\mathrm{in} 1 t}+L_{\mathrm{in} 2 t}+X_{t} \times G_{t-1}\right)$,

where $L_{\text {in } 1 t}$ and $L_{\text {in2t }}$ represent the input source language, $X_{t}$ is the logic of $V_{\mathrm{G}}$ at the current moment, and $G_{t-1}$ is the conductance generated by the previous input. So the current conductance is not only dependent on the current input, but also the conductance at the previous moment, and $X_{t}$ can control whether to forget the information of the previous moment, and detailed process at each time is shown in Fig. S18. The output of two MOSTs after post-processing is converted into a voltage signal and passed to the next basic unit. For simplicity, the LSTM layer is composed of 6 MOSTs (Fig. 5d), and associative memory implemented on this MOST-based LSTM layer can convert English to Chinese, such as those from AI Challenger (more information about the dataset is provided in the Supplementary information S2). In the training stage, the text of English is converted into a light sequence according to the conversion tables presented in Table S2, and is fed to the LSTM. As mentioned, $X_{t}$ (the time series of $V_{\mathrm{GS}}$ ) controls whether to memorize the input at the previous moment, and the overall output is obtained from the last time (Fig. S18). The output is passed to the softmax layer and the classification layer, and finally the target language is obtained. In the recall stage, the source language is encoded as a light sequence, which is fed into the MOST-based LSTM layer as input signals. And $X_{t}$ decides whether the context is relevant, which means that different target language outputs are controlled by $X_{t}$ with the same source language input. As shown in Fig. $5 \mathrm{e}$, in the case of the same input, the target language with one or two words can be obtained by control of $X_{t}$.

\section{CONCLUSIONS}

In summary, for the first time, a multilevel photosensitive blending MOST is invented, which exhibits 8 stable and well distinguishable states, and synaptic behaviors such as EPSC, STM, and LTM are successfully mimicked. The shallow trap center caused by discontinuous n-type quantum and the deep trap center caused by QDs promise MOST with different synaptic states under light illuminations, which return to the same state after removing the light. Based on MOST, an ODM is demonstrated, which is the first application of neuromorphic device to the field of NMT. It replaces traditional four sigmoid layers by a single-layer network composed of MOST with the characteristic of "long-term dependence", and significantly simplifies the structure of traditional NMT system. Moreover, MOST with multiple synaptic levels controlled by light can significantly reduce the number of components and the power consumption of the codec model, alleviating the problems caused by excessive data. Hence, this work applies neuromorphic devices in NMT to reduce the system's energy consumption rate, and proposes a new multi-level synaptic transistor as the based cell of decoding module to improve the data utilization rate, which will lay the foundation for breaking the bottleneck of machine translation.

\section{Received 23 September 2021; accepted 16 November 2021; published online 12 January 2022}

1 Castelfranchi C. Alan turing's "computing machinery and intelligence". Topoi, 2013, 32: 293-299

2 Junczys-Dowmunt M, Dwojak T, Hoang H. Is neural machine trans- lation ready for deployment? A case study on 30 translation directions. 2016, arXiv: abs/1610.01108

3 Bahdanau D, Cho K, Bengio Y. Neural machine translation by jointly learning to align and translate. 2014, arXiv:1409.0473

4 Sutskever I, Vinyals O, Le QV. Sequence to sequence learning with neural networks. Adv Neur In, 2014, 27

5 Ignatoski M, Lerga J, Stanković L, et al. Comparison of entropy and dictionary based text compression in English, German, French, Italian, Czech, Hungarian, Finnish, and Croatian. Mathematics, 2020, 8: 1059

6 Lawnik M, Pełka A, Kapczyński A. A new way to store simple text files. Algorithms, 2020, 13: 101

7 Bengio Y, Senecal JS. Adaptive importance sampling to accelerate training of a neural probabilistic language model. IEEE Trans Neural Netw, 2008, 19: 713-722

8 Jain S, Venkataramani S, Srinivasan V, et al. Compensated-DNN: Energy efficient low-precision deep neural networks by compensating quantization errors. In: 2018 55th Acm/Esda/Ieee Design Automation Conference (DAC). San Francisco, 2018

$9 \mathrm{Xu} \mathrm{H}, \mathrm{Li} \mathrm{K}$, Wang $\mathrm{Y}$, et al. Neural network language modeling with letter-based features and importance sampling. In: 2018 IEEE International Conference on Acoustics, Speech and Signal Processing (ICASSP). Calgary, 2018, 6109-6113

10 Sennrich R, Haddow B, Birch A. Improving neural machine translation models with monolingual data. 2016, arXiv:1511.06709

11 Ielmini D, Wong HSP. In-memory computing with resistive switching devices. Nat Electron, 2018, 1: 333-343

12 Waldrop MM. The chips are down for Moore's law. Nature, 2016, 530: 144-147

13 Misra J, Saha I. Artificial neural networks in hardware: A survey of two decades of progress. Neurocomputing, 2010, 74: 239-255

14 Yang S, Deng B, Wang J, et al. Scalable digital neuromorphic architecture for large-scale biophysically meaningful neural network with multi-compartment neurons. IEEE Trans Neural Netw Learn Syst, 2020, 31: 148-162

15 Wong HSP, Salahuddin S. Memory leads the way to better computing. Nat Nanotech, 2015, 10: 191-194

16 Yao P, Wu H, Gao B, et al. Fully hardware-implemented memristor convolutional neural network. Nature, 2020, 577: 641-646

17 Melianas A, Quill TJ, LeCroy G, et al. Temperature-resilient solid-state organic artificial synapses for neuromorphic computing. Sci Adv, 2020, 6: eabb2958

18 Wang S, Liu L, Gan L, et al. Two-dimensional ferroelectric channel transistors integrating ultra-fast memory and neural computing. Nat Commun, 2021, 12: 53

19 Prezioso M, Merrikh-Bayat F, Hoskins BD, et al. Training and operation of an integrated neuromorphic network based on metal-oxide memristors. Nature, 2015, 521: 61-64

20 Luo S, Liao K, Lei P, et al. A synaptic memristor based on twodimensional layered $\mathrm{WSe}_{2}$ nanosheets with short- and long-term plasticity. Nanoscale, 2021, 13: 6654-6660

21 Kuzum D, Jeyasingh RGD, Lee B, et al. Nanoelectronic programmable synapses based on phase change materials for brain-inspired computing. Nano Lett, 2012, 12: 2179-2186

22 Sebastian A, Le Gallo M, Khaddam-Aljameh R, et al. Memory devices and applications for in-memory computing. Nat Nanotechnol, 2020, 15: $529-544$

23 Munoz-Martin I, Bianchi S, Pedretti G, et al. Unsupervised learning to overcome catastrophic forgetting in neural networks. IEEE J Explor Solid-State Comput Devices Circuits, 2019, 5: 58-66

24 Huang J, Chen J, Yu R, et al. Tuning the synaptic behaviors of biocompatible synaptic transistor through ion-doping. Org Electron, 2021, 89: 106019

25 Ke Y, Yu R, Lan S, et al. Polymer bulk-heterojunction synaptic fieldeffect transistors with tunable decay constant. J Mater Chem C, 2021, 9: 4854-4861

26 Lan S, Zhong J, Chen J, et al. An optoelectronic synaptic transistor with efficient dual modulation by light illumination. J Mater Chem C, 2021, 9: $3412-3420$

27 Lee Y, Park HL, Kim Y, et al. Organic electronic synapses with low 
energy consumption. Joule, 2021, 5: 794-810

28 Lu K, Li X, Sun Q, et al. Solution-processed electronics for artificial synapses. Mater Horiz, 2021, 8: 447-470

29 Yang CS, Shang DS, Liu N, et al. All-solid-state synaptic transistor with ultralow conductance for neuromorphic computing. Adv Funct Mater, 2018, 28: 1804170

30 Chen J, Li E, Yan Y, et al. Flexible metal oxide synaptic transistors using biomass-based hydrogel as gate dielectric. J Phys D-Appl Phys, 2019, 52: 484002

31 Li E, Lin W, Yan Y, et al. Synaptic transistor capable of accelerated learning induced by temperature-facilitated modulation of synaptic plasticity. ACS Appl Mater Interfaces, 2019, 11: 46008-46016

32 Ren Y, Yang X, Zhou L, et al. Recent advances in ambipolar transistors for functional applications. Adv Funct Mater, 2019, 29: 1902105

33 Liu Y, Li E, Wang X, et al. Self-powered artificial auditory pathway for intelligent neuromorphic computing and sound detection. Nano Energy, 2020, 78: 105403

34 Liu Y, Yang W, Yan Y, et al. Self-powered high-sensitivity sensory memory actuated by triboelectric sensory receptor for real-time neuromorphic computing. Nano Energy, 2020, 75: 104930

35 Zang Y, Shen H, Huang D, et al. A dual-organic-transistor-based tactile-perception system with signal-processing functionality. Adv Mater, 2017, 29: 1606088

36 Pradhan B, Das S, Li J, et al. Ultrasensitive and ultrathin phototransistors and photonic synapses using perovskite quantum dots grown from graphene lattice. Sci Adv, 2020, 6: eaay5225

$37 \mathrm{Yu} \mathrm{R}, \mathrm{Li} \mathrm{E}, \mathrm{Wu} \mathrm{X}$, et al. Electret-based organic synaptic transistor for neuromorphic computing. ACS Appl Mater Interfaces, 2020, 12: 15446-15455

38 Kim MK, Lee JS. Ferroelectric analog synaptic transistors. Nano Lett, 2019, 19: 2044-2050

39 Cho K, van Merrienboer B, Gulcehre C, et al. Learning phrase representations using RNN encoder-decoder for statistical machine translation. 2014, arXiv:1406.1078

40 Gao L, Li X, Song J, et al. Hierarchical LSTMs with adaptive attention for visual captioning. IEEE Trans Pattern Anal Mach Intell, 2019, 42: 1

41 Zheng Y, Yang HG, Huang ZH, et al. A high energy-efficiency FPGAbased LSTM accelerator architecture design by structured pruning and normalized linear quantization. In: 2019 International Conference on Field-Programmable Technology (ICFPT 2019). Tianjin, 2019, 271-274

42 Hochreiter S, Schmidhuber J. Long short-term memory. Neural Comput, 1997, 9: 1735-1780

Acknowledgements This work was supported by the National Natural Science Foundation of China (61974029), the Natural Science Foundation for Distinguished Young Scholars of Fujian Province (2020J06012), and Fujian Science \& Technology Innovation Laboratory for Optoelectronic Information of China (2021ZZ129).

Author contributions Chen $\mathrm{H}$ and Guo $\mathrm{T}$ conceived the project; Zhang X, $\mathrm{Li} \mathrm{E}$ and $\mathrm{Yu} \mathrm{W}$ designed and performed the experiments and collected the data; Zhang X, Yu R, He L, and Li E analyzed and discussed the data; Zhang $\mathrm{X}$ and Chen $\mathrm{H}$ wrote the paper; All authors contributed to the general discussion.

Conflict of interest The authors declare that they have no conflict of interest.

Supplementary information Experimental details and supporting data are available in the online version of the paper.

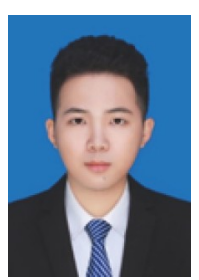

Xianghong Zhang got his bachelor's degree from the College of Physics and Information Engineering, Fuzhou University in 2019. Currently, he is a PhD candidate under the supervision of Prof. Huipeng Chen at the College of Physics and Information Engineering, Fuzhou University. His current research interests mainly focus on the neuromorphic devices (synaptic device and neuron device).

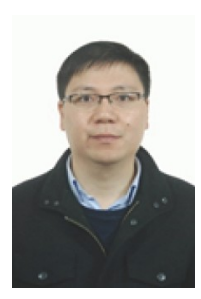

Huipeng Chen got his $\mathrm{PhD}$ degree in physics from Tufts University in 2009. Before joining the College of Physics and Information Engineering, Fuzhou University in 2015, he worked as a postdoctoral fellow at Texas Tech University during 2009-2011 and the University of Tennessee and Oak Ridge National Laboratory from 2011 to 2014 His research interest focuses on semiconductor materials and devices, including thin film transistors, memories, sensors, neuromorphic electronic devices and systems.

\section{用于神经机器翻译的浮栅型PN共混光电突触晶体管}

张翔鸿 ${ }^{1,2}$, 李恩龙 ${ }^{1,2}$, 俞社坚 ${ }^{1,2}$, 何立铧 ${ }^{1,2}$, 余伟杰 ${ }^{1,2}$, 陈惠鹏 ${ }^{1,2 *}$, 郭太良 ${ }^{1,2}$

摘要 具有编码器-解码器框架的神经机器翻译被认为是未来机器翻译 的一种主要框架. 然而, 由于多种语言融合和新词不断涌现, 目前大多 数基于冯诺依曼架构的神经机器翻译系统的解码器设备数量大幅增 加, 导致系统功耗过高. 本研究首先展示了具有两种不同捕获机制的多 级光敏混合半导体光电突触晶体管(MOST), 它在480-800 nm波长范围 的光照下表现出 8 种稳定且易于区分的状态和突触行为, 且均具备兴奋 性突触后电流、短期记忆和长期记忆等突触性能. 此外, 本研究首次将 神经形态器件应用在神经机器翻译领域, 成功制作了基于MOST的光解 码器模型, 显著简化了传统神经机器翻译系统的结构. 并且作为一种多 级突触器件, MOST可以进一步减少器件数量, 在光照下简化编解码模 型的结构. 这项研究首先将神经形态器件应用于神经机器翻译, 并提出 了一种多级突触晶体管作为解码模块的基础单元, 为打破机器翻译的 瓶颈奠定了坚实的基础. 